\title{
ANALYSIS OF AN $M|G| 1 \mid R$ QUEUE WITH BATCH ARRIVALS AND TWO HYSTERETIC OVERLOAD CONTROL POLICIES
}

\author{
Yuliya GAidAMAKA*, Alexander PECHINKIN **, Rostislav RAZUMCHIK **,*, \\ KONSTANTIN SAMOUYLOV*, EDUARD SOPIN* \\ * Department of Applied Probability and Informatics \\ Peoples' Friendship University of Russia, Miklukho-Maklaya str., 6, Moscow 117198, Russia \\ e-mail: \{ygaidamaka, ksam\} @sci.pfu.edu.ru, sopin-eduard@yandex.ru \\ ${ }^{* *}$ Institute of Problems of Informatics \\ Russian Academy of Sciences, Vavilova str., 44-2, Moscow 119333, Russia \\ e-mail: apechinkin@ipiran.ru
}

\begin{abstract}
Hysteretic control of arrivals is one of the most easy-to-implement and effective solutions of overload problems occurring in SIP-servers. A mathematical model of an SIP server based on the queueing system $M^{[X]}|G| 1\langle L, H\rangle \mid\langle H, R\rangle$ with batch arrivals and two hysteretic loops is being analyzed. This paper proposes two analytical methods for studying performance characteristics related to the number of customers in the system. Two control policies defined by instants when it is decided to change the system's mode are considered. The expression for an important performance characteristic of each policy (the mean time between changes in the system mode) is presented. Numerical examples that allow comparison of the efficiency of both policies are given.
\end{abstract}

Keywords: queueing system, batch arrival, general service time, overload, hysteretic load control.

\section{Introduction}

The problem of overload protection of essential components of a telecommunications network has again become crucial in $3 \mathrm{G}$ and $4 \mathrm{G}$ generation networks. At this time, the reliability of a network is compromised by a rapid growth in the number of telecommunications services, characterized by unpredictable traffic transmitted over a network, and the high performance requirements for network nodes and servers. The problem's manifestations were, for example, various kinds of SIP-server (Session Initiation Protocol) overload generated by avalanche flood of requests from users for broadband services (Hilt et al., 2011). For example, in the simplest case, voice-over-IP service, a user request requires transfer and processing by multiple servers at least seven SIP-messages. The explosive nature of the traffic, whose processing is not handled by the most modern equipment, is not only the behavior of people in the busy hours, as previously in $2 \mathrm{G}$ circuit switched networks, but also due to a variety of other reasons. The most typical causes of SIP-server overload such as poor capacity planning, component failures, flash crowds, denial of service attacks are described in documents of the Internet Engineering Task Force (IETF), which are considered international standards for IP networks. One of the most problematic sources of overload is avalanche restart, when a large number of users simultaneously attempt to register on SIP-servers. An example is the so-called "Manhattan reboots" scenario, when an outage accident happened in the largest area of the city, and after the power was restored, all SIP-terminals simultaneously tried to log on to servers, thus creating a large flow of REGISTER SIP-messages. The problem considered in the paper relates to control theory and performance analysis of complex technical systems (Kaczorek, 2013; Olwal et al., 2012; Moltchanov et al., 2006).

In fact, it is required to solve two problems: how to define the beginning of overload and how to eliminate it. The most obvious solution is the introduction of queue length thresholds, just as is done in the ITU-T recommendations for network and link layers of the common channel signaling system no. 7 (SS7) (Russell, 
2006).

Abaev et al. (2011; 2012b; 2012a) give a general overview and analysis of the hysteretic load control mechanism used in SS7, and the mathematical model developed for local overload control in an SIP-server network. Overload control is carried out by introducing three thresholds in the queue of messages waiting to be processed in the buffer of a server. They are overload onset threshold $H$, overload abatement threshold $L$, and load discard threshold $R$. The server operates in one of three modes: normal, overload, and discard, each with its own arrival rate. At that, the behavior of the server differs: the control system acts to reduce the input traffic by discarding some of the input messages in overload mode and all the input messages in discard mode. While the total number of messages in the queue does not exceed the onset threshold $H$, the server is considered to be operating in normal mode. If the queue length becomes equal to $H$, the control system switches to overload mode in order to eliminate overload. The server remains in this mode until the queue length falls to the abatement threshold $L$ in case of the mitigating of overload or until the queue length overtakes $(R-1)$. After the queue length drops below $L$, the control system detects elimination of overload and switches to normal mode. If the queue length increases to $R$, the control system detects aggravation of overload and switches to discard mode. If in discard mode the queue length drops below $H$, the control system switches to overload mode. Threshold values are chosen so that $0<L<H<R$, leading to the so-called hysteretic effect (Gebhart, 1967; Krasnoselskii and Pokrovskii, 1989) between the pairs of thresholds $\langle L, H\rangle$ and $\langle H, R\rangle$ in the form of two loops-one for each pair of thresholds, as shown in Fig. 1. In SS7 protocols, hysteretic control has been introduced (Takshing and Yen, 1983; Brown et al., 1984) to reduce the number of control system switchings between operating modes. At that, the problem was solved by the choice of thresholds in order to minimize the mean return time of the system from overload states to normal load states.

In many papers devoted to the study of queueing

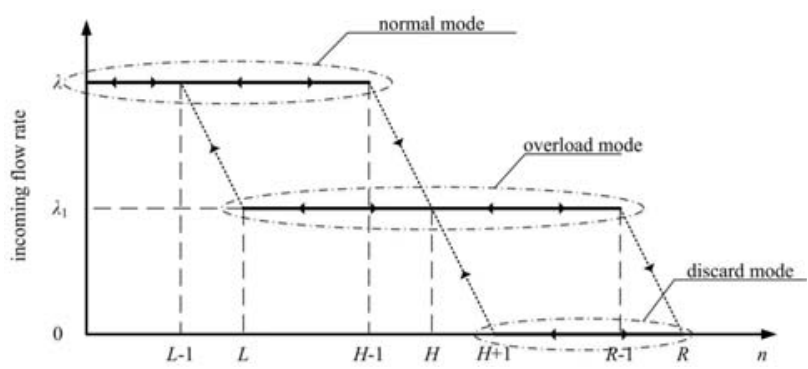

Fig. 1. Hysteretic load control. systems with hysteresis control more common are the models with hysteretic service disciplines (Golubchik and Lui, 1997; Sindal and Tokekar, 2008), and less so the models with hysteretic input flow (Takagi, 1985; Benaboud and Mikou, 2002). One can find a comprehensive review of results on the hysteresis control in the work of Dshalalow (1997) and Bekker (2009). The closest to our research model and methods are those developed by Roughan and Pearce (2000), who give numerous references on the problem of the analysis of queueing systems with hysteresis control of the incoming flow intensity (hereinafter-with hysteretic load control). Abaev et al. (2011; 2012b; 2012a) as well as Pechinkin and Razumchik (2013a; 2013b) review papers on mathematical modeling and simulation of systems with hysteretic load control. The queueing system with Poisson input, exponential and general service times was built there and thoroughly studied. The parameter of interest from the viewpoint of SIP-server performance is the total time spent by the server in overload and discard modes. This random variable is called the return time of the system from the overload states to the normal load states. Its characteristics such as the mean value and the $95 \%$ quantile are subject to minimization with the given constraints on the load and structural parameters of the system. In the work of Abaev et al. (2014) for the Markovian case, an algorithm was developed for calculation of the mean return time and the problem of its minimization was solved numerically for practically important values of the model parameters.

The contribution of this paper is the mathematical model for SIP-server overload control-queueing system $M^{[X]}|G| 1\langle L, H\rangle \mid\langle H, R\rangle$ with hysteretic load control and batch arrivals - and methods of its stationary analysis. We consider two control policies based on the time instants when switching between operating modes occurs. According to policy P1, the system may switch between operating modes at time instants when any change in the number of customers in the system (arrival or departure) occurs; according to policy P2-only at time instants of service completion.

Our main result is Theorem 1, which gives the equation for the stationary probability distribution of the system states with hysteresis policy $\mathrm{P} 1$. We also prove Theorem 2, which gives a method of calculating the stationary distribution of the queue length for the system with control policy P2.

The paper is organized as follows. In Section 2 we describe in detail the proposed model and introduce all necessary concepts and denotation. In Sections 3 and 4 we carry out the mathematical analysis of the model for control policies P1 and P2. In Section 5 examples of numerical analysis are provided, key results are summarized and directions of further research are given. 


\section{System description}

Consider a single-queue, single-server system with a general service time distribution function which is denoted by $B(x)$ and hysteretic load control. By $\beta(s)$ we denote a Laplace-Stieltjes Transform (LST) of $B(x)$, and by $b$ the mean service time. In addition, we use the notation

$$
\tilde{\beta}(s)=\int_{0}^{\infty} e^{-s x}[1-B(x)] \mathrm{d} x=\frac{1}{s}[1-\beta(s)] .
$$

Two types of customers (say, type 1 and type 2) arrive at the system in batches (each) in accordance with a time-homogeneous Poisson process with rates $\lambda_{1}$ and $\lambda_{2}$, respectively. Henceforth $\lambda=\lambda_{1}+\lambda_{2}$. Each arriving batch has a random number of customers and the probability that the arriving batch of type $k, k=1,2$, customers contains exactly $n, n \geq 1$, customers is $\omega_{k, n}$. Write

$$
\begin{gathered}
\Omega_{k, n}=\sum_{m=n}^{\infty} \omega_{k, m}, \quad k=1,2, \quad n \geq 1, \\
\omega_{n}=\frac{\lambda_{1} \omega_{1, n}+\lambda_{2} \omega_{2, n}}{\lambda}, \quad n \geq 1, \\
\Omega_{n}=\sum_{m=n}^{\infty} \omega_{m}, \quad n \geq 1 .
\end{gathered}
$$

Clearly, $\Omega_{k, n}, \quad k=1,2, n \geq 1$, is the probability that there are at least $n$ customers in the arriving batch of type $k$ customers, whereas $\omega_{n}, n \geq 1$, is the probability that there are exactly $n$ customers in the batches of any type customers batches arriving at the system. Finally, $\Omega_{n}$, $n \geq 1$, is the probability that there are at least $n$ customers in the batches of any type customers batches arriving at the system.

Customers are served one at a time. In this section we are only interested in a stationary queue length distribution and related performance characteristics. Thus we do not specify the order of service for batches and inside batches as well as a discard rule when only part of a batch can be accepted by the system.

The hysteretic load control is functioning as follows. The server operates in three modes: normal $(s=0)$, overload $(s=1)$, and discard $(s=2)$, where $s$ is the overload status (see Fig. 1). From the time instant of the first customer's arrival into the system until the time instant when the number of customers increases to $H$, the system is considered to be in normal mode, where all incoming customers are taken into the system. When the number of customers in the system becomes no less than $H$, the system switches to overload mode, where only incoming type 1 customers are taken into the system, and incoming type 1 customers are blocked. The system is considered to be in overload mode until the number of customers in the system drops to $(L-1)$ or until the number of customers overtakes $(R-1)$. In the first case, the system switches to normal mode, where all incoming customers are taken into the system. In the second case the system switches to discard mode, where all incoming customers are blocked, and stays in discard mode until the number of customers drops to $H$. Then the procedure continues.

We assume that the condition $b<\infty$ holds for the system which is necessary and sufficient for the existence of a steady state.

In further analysis it is assumed that threshold values are chosen in such a way that inequalities $H-L \geq 1$ and $R-H \geq 2$ hold. Thus, special cases are left out of scope. This is done only for convenience because computational expressions for them differ from those presented below (are simpler and thus omitted).

Throughout the paper, in order to present expressions in a concise form, we use the agreement that $\sum_{j=i}^{i-1} x_{j}=0$ and $\prod_{j=i}^{i-1} x_{j}=1$.

\section{Policy P1}

In this section we obtain a stationary queue length distribution in an $M^{[X]}|G| 1\langle L, H\rangle \mid\langle H, R\rangle$ system operating under policy $\mathrm{P} 1$ and give a computational expression for its several important performance characteristics.

Note that policy P1 implies that the system changes its state from normal mode to overload mode at the moment when a batch of customers arrives at the system and the total number of customers in the system jumps to the value between $H$ and $(R-1)$. Similarly, the system changes its state from normal or overload mode to discard mode if the size of the arriving batch exceeds the system's current free capacity. Those customers in the batch that find room in the queue stay in the system and the rest of them leave it without having any further effect on it (they are considered to be lost).

In the next subsection, auxiliary functions significant for further analysis are introduced. Then, using the supplementary variable method and the state space elimination method, we obtain the stationary distribution of the Markov process describing the system's behaviour. In the closing subsection we give expressions for the probability that the system is in normal/overload/discard mode, the stationary mean control cycle time and the mean time the system spends in overload and discard modes.

Throughout this section, for the sake of simplicity, we assume that there exists a density $b(x)=B^{\prime}(x)$.

3.1. Auxiliary functions. Assume that at an arbitrary time instant the total number of customers in the system 
is $n, n=\overline{H+1, R-1}$, the system is in overload mode (i.e., only batches of type 1 customers are allowed to enter system) and the elapsed service time of the customer in service is $x$. Denote by $\alpha_{n}(x)$ the probability that until the time instant when the total number of customers in the system equals $(n-1)$ for the first time, there will never be $R$ customers in the system (or, alternatively, the total number of customers in the system will reach $(n-1)$ earlier than $R$ ).

Write

$$
q_{n}(x)=[1-B(x)] \alpha_{n}(x), \quad n=\overline{H+1, R-1} .
$$

It can be shown that, for functions $q_{n}(x), n=$ $\overline{H+1, R-1}$, the following equations hold:

$$
\begin{aligned}
-q_{n}^{\prime}(x)= & -\lambda_{1} q_{n}(x)+b(x) \\
& +\lambda_{1} \sum_{m=1}^{R-1-n} \omega_{1, m} q_{n+m}(x) \prod_{i=0}^{m-1} \alpha_{n+i}(0) .
\end{aligned}
$$

Solving (2) for $n=R-1$ yields

$$
q_{R-1}(x)=C e^{\lambda_{1} y}+\int_{x}^{\infty} b(y) e^{-\lambda_{1}(y-x)} \mathrm{d} y,
$$

where $C$ is a constant. Since $\alpha_{R-1}(x)$ is a probability for each value of $x$, using the property $\left|\alpha_{R-1}(x)\right| \leq 1$, one finds that $C=0$. Thus

$$
q_{R-1}(x)=\int_{x}^{\infty} b(y) e^{-\lambda_{1}(y-x)} \mathrm{d} y .
$$

In the same manner, one can obtain from (2) the following expression for $q_{n}(x), n=\overline{H+1, R-2}$ :

$$
\begin{aligned}
q_{n}(x)= & \int_{x}^{\infty}\left[b(y)+\lambda_{1} \sum_{m=1}^{R-1-n} \omega_{1, m} q_{n+m}(y)\right. \\
& \left.\times \prod_{i=0}^{m-1} \alpha_{n+i}(0)\right] e^{-\lambda_{1}(y-x)} \mathrm{d} y .
\end{aligned}
$$

The latter expression contains unknown quantities $\alpha_{n}(0)$. Setting $x=0$ in (3) and (4), and taking into account (1), one can find them in the form

$$
\begin{aligned}
\alpha_{n}(0)= & \left(1-\lambda_{1} \sum_{m=1}^{R-1-n} \omega_{1, m} \int_{0}^{\infty} q_{n+m}(y) e^{-\lambda_{1} y} \mathrm{~d} y\right. \\
& \left.\times \prod_{i=1}^{m-1} \alpha_{n+i}(0)\right)^{-1} \beta\left(\lambda_{1}\right), \\
n=\overline{H+1, R-1} &
\end{aligned}
$$

Now assume that at an arbitrary time instant the total number of customers in the system is $n, n=\overline{L, H-1}$, the system is in normal mode and the elapsed service time of the customer in service is $x$. Denote by $\alpha_{n}(x)$ the probability that, until the time instant when the total number of customers in the system becomes equals to $(n-$ 1) for the first time, there will never be $H$ customers in the system (or, alternatively, the total number of customers in the system will reach $(n-1)$ earlier than $H)$.

If we write

$$
q_{n}(x)=[1-B(x)] \alpha_{n}(x), \quad n=\overline{L, H-1},
$$

and use the same argument as above, it can be shown that probabilities $q_{n}(x), n=\overline{L, H-1}$ can be computed using the following expressions:

$$
\begin{aligned}
q_{n}(x) & \\
= & \int_{x}^{\infty}\left[b(y)+\lambda \sum_{m=1}^{H-1-n} \omega_{m} q_{n+m}(y)\right. \\
& \left.\times \prod_{i=0}^{m-1} \alpha_{n+i}(0)\right] e^{-\lambda(y-x)} \mathrm{d} y, \quad n=\overline{L, H-1}
\end{aligned}
$$

$$
\begin{aligned}
& \alpha_{n}(0) \\
& =\left(1-\lambda \sum_{m=1}^{H-1-n} \omega_{m} \int_{0}^{\infty} q_{n+m}(y) e^{-\lambda y} \mathrm{~d} y\right. \\
& \left.\quad \times \prod_{i=1}^{m-1} \alpha_{n+i}(0)\right)^{-1} \beta(\lambda), \quad n=\overline{L, H-1} .
\end{aligned}
$$

For numerical computation it is more convenient to transform the expressions obtained above in the following way. and

Introduce functions $Q_{0}(x)=\tilde{Q}_{0}(x)=b(x), x>0$,

$$
\tilde{Q}_{n}(x)=\int_{x}^{\infty} \tilde{Q}_{n-1}(y) e^{-\lambda_{1}(y-x)} \mathrm{d} y, \quad n=\overline{1, R-H}
$$

$$
Q_{n}(x)=\int_{x}^{\infty} Q_{n-1}(y) e^{-\lambda(y-x)} \mathrm{d} y, \quad n=\overline{1, H-L} .
$$

Through careful inspection of probabilities $q_{R-n}(x)$, $n=\overline{1, R-H-1}$, one can find that they can be rewritten in an alternative form, i.e.,

$$
q_{R-n}(x)=\sum_{k=1}^{n} \tilde{d}_{n, k} \tilde{Q}_{k}(x), \quad n=\overline{1, R-H-1} .
$$


Then from (3) we have $q_{R-1}(x)=\tilde{Q}_{1}(x)$ and $\tilde{d}_{1,1}=1$. Further, from (4) it follows that

$$
\begin{gathered}
q_{R-n}(x)=\tilde{Q}_{1}(x)+\lambda_{1} \sum_{m=1}^{n-1} \omega_{1, m} \sum_{k=1}^{n-m} \tilde{d}_{n-m, k} \\
\times \tilde{Q}_{k+1}(x) \prod_{i=0}^{m-1} \alpha_{R-n+i}(0), \\
n=\frac{2, R-H-1 .}{2, R}
\end{gathered}
$$

Thus we obtain coefficients $\tilde{d}_{n, k}$ in the form

$$
\begin{array}{r}
\tilde{d}_{n, k}=\lambda_{1} \sum_{m=1}^{n-k+1} \omega_{1, m} \tilde{d}_{n-m, k-1} \prod_{i=0}^{m-1} \alpha_{R-n+i}(0), \\
n=\overline{2, R-H-1}, \quad k=\overline{2, n .}
\end{array}
$$

In particular, for $n=\overline{1, R-H-1}$, it holds that

$$
\int_{0}^{\infty} q_{R-n}(x) e^{-\lambda_{1} x} \mathrm{~d} x=\sum_{k=1}^{n} \tilde{d}_{n, k} \tilde{Q}_{k+1}(0) .
$$

A similar argument leads to the following result for probabilities $q_{H-n}(x), n=\overline{1, H-L}$. Let

$$
q_{H-n}(x)=\sum_{k=1}^{n} d_{n, k} Q_{k}(x), \quad n=\overline{1, H-L} .
$$

Then from (6) for $n=H-1$ we get $q_{H-1}(x)=Q_{1}(x)$, thus $d_{1,1}=1$. Similarly, from (6) for $n=\overline{L, H-2}$ one can verify that coefficients $d_{n, k}, n=\overline{2, H-L}, k=\overline{2, n}$ are given by

$$
d_{n, k}=\lambda \sum_{m=1}^{n-k+1} \omega_{m} d_{n-m, k-1} \prod_{i=0}^{m-1} \alpha_{H-n+i}(0) .
$$

Notice that

$$
\int_{0}^{\infty} q_{H-n}(x) e^{-\lambda x} \mathrm{~d} x=\sum_{k=1}^{n} d_{n, k} Q_{k+1}(0), \quad n=\overline{1, H-L} .
$$

3.2. Stationary distribution. Having introduced all necessary auxiliary functions, we turn to the derivation and solution of equations for the stationary probability distribution.

Denote by $p_{n}(x), \quad n=\overline{1, H-1}$, the stationary probability density that the total number of customers in the system is $n$, the elapsed service time of a currently served customer is $x$ and the system is in normal mode (i.e., batches of both types of customers are allowed to enter the system); $p_{n}(x), n=\overline{H, R-1}$, the stationary probability density that the total number of customers in the system is $n$, elapsed service time of a currently served customer is $x$ and the system is in overload mode (i.e. only batches of type 1 customers are allowed to enter the system); by $\tilde{p}_{n}(x), n=\overline{L, H-1}$, the stationary probability density, that the total number of customers in the system is $n$, the elapsed service time of a currently served customer is $x$ and the system is in overload mode; by $\tilde{p}_{n}(x), \quad n=\overline{H+1, R}$, the stationary probability density that the total number of customers in the system is $n$, the elapsed service time of a currently served customer is $x$ and the system is in discard mode (i.e., none of the arriving customers are allowed to enter the system).

Let also $p_{n}$ and $\tilde{p}_{n}$ denote stationary probabilities of $n$ customers in the system, i.e.,

$$
\begin{aligned}
p_{n} & =\int_{0}^{\infty} p_{n}(x) \mathrm{d} x, \quad n=\overline{1, R-1}, \\
\tilde{p}_{n} & =\int_{0}^{\infty} \tilde{p}_{n}(x) \mathrm{d} x, \quad n=\overline{L, R}, \quad n \neq H .
\end{aligned}
$$

3.2.1. Computation of $p_{n}(x)$ for $n=\overline{1, L-1}$. Let us start with the derivation of equations for stationary probability densities $p_{n}(x), n=\overline{1, L-1}$. Introduce new functions $r_{n}(x)$ defined by the relation

$$
p_{n}(x)=[1-B(x)] r_{n}(x), \quad n=\overline{1, L-1} .
$$

By writing out differential equations for $p_{n}(x)$ and using the substitution (10), after some simple transformations one can verify that the following equation holds:

$$
r_{n}^{\prime}(x)=-\lambda r_{n}(x)+\lambda \sum_{m=1}^{n-1} \omega_{n-m} r_{m}(x), \quad n=\overline{1, L-1},
$$

whose solution for $n=\overline{1, L-1}$ has the form

$$
\begin{aligned}
& r_{n}(x) \\
& =e^{-\lambda x}\left(c_{n, n}+\lambda \sum_{m=1}^{n-1} \omega_{n-m} \int_{0}^{x} e^{\lambda y} r_{m}(y) \mathrm{d} y\right) .
\end{aligned}
$$

Solving (11) for $n=1,2$ and then using induction, one readily gets

$$
r_{n}(x)=e^{-\lambda x} \sum_{k=0}^{n-1} \frac{(\lambda x)^{k}}{k !} c_{n, n-k}, \quad n=\overline{1, L-1} .
$$

Substitution of (13) into (12) leads to the expression for $r_{n}(x), n=\overline{1, L-1}$ :

$$
\begin{aligned}
& r_{n}(x) \\
& =e^{-\lambda x}\left(c_{n, n}+\sum_{m=1}^{n-1} \omega_{n-m} \sum_{k=0}^{m-1} \frac{(\lambda x)^{k+1}}{(k+1) !} c_{m, m-k}\right),
\end{aligned}
$$


wherefrom recurrence relations for coefficients $c_{n, m}$ follow. That is, for $n=\overline{2, L-1}, m=\overline{1, n-1}$,

$$
c_{n, n-m}=\sum_{k=m}^{n-1} \omega_{n-k} c_{k, k-m+1} .
$$

In order to determine the only unknown term $c_{n, n}$ in (14), we require certain additional (boundary) conditions. Removing from consideration all time instants when the total number of customers in the system is greater than $n$ and using the elimination method, one can come to the following equation:

$$
\begin{aligned}
c_{n, n}= & r_{n}(0)=p_{n}(0) \\
= & \lambda \Omega_{n} p_{0}+\lambda \sum_{i=1}^{n} \Omega_{n-i+1} p_{i} \\
= & \lambda \Omega_{n} p_{0}+\lambda \sum_{i=1}^{n-1} \Omega_{n-i+1} p_{i}+\lambda \int_{0}^{\infty}[1-B(x)] r_{n}(x) \mathrm{d} x \\
= & \lambda \Omega_{n} p_{0}+\lambda \sum_{i=1}^{n-1} \Omega_{n-i+1} p_{i} \\
& +\lambda \int_{0}^{\infty}[1-B(x)] e^{-\lambda x} \sum_{k=0}^{n-1} \frac{(\lambda x)^{k}}{k !} c_{n, n-k} \mathrm{~d} x \\
= & \lambda \Omega_{n} p_{0}+\lambda \sum_{i=1}^{n-1} \Omega_{n-i+1} p_{i} \\
& +\lambda\left(\beta_{0} c_{n, n}+\sum_{k=1}^{n-1} \beta_{k} c_{n, n-k}\right), \quad n=\overline{1, L-1},
\end{aligned}
$$

where

$$
\begin{aligned}
\beta_{k} & =\frac{\lambda^{k}}{k !} \int_{0}^{\infty} x^{k} e^{-\lambda x}[1-B(x)] \mathrm{d} x \\
& =\frac{(-\lambda)^{k}}{k !} \tilde{\beta}^{(k)}(\lambda) .
\end{aligned}
$$

Here and henceforth by $\tilde{\beta}^{(k)}(s), \quad k \geq 0$, we denote the $k$-th derivative of $\tilde{\beta}(s)$ with respect to $s$. Solution of the latter equation yields the following expression for $c_{n, n}$ :

$$
\begin{aligned}
c_{n, n}= & \lambda\left(1-\lambda \beta_{0}\right)^{-1}\left(\Omega_{n} p_{0}+\sum_{i=1}^{n-1} \Omega_{n-i+1} p_{i}\right. \\
& \left.+\sum_{k=1}^{n-1} \beta_{k} c_{n, n-k}\right), \quad n=\overline{1, L-1} .
\end{aligned}
$$

Thus the probability that the number of customers in the system is $n, n=\overline{1, L-1}$ (and it is in normal mode), is given by

$$
\begin{aligned}
p_{n} & =\int_{0}^{\infty}[1-B(x)] e^{-\lambda x} \sum_{k=0}^{n-1} \frac{(\lambda x)^{k}}{k !} c_{n, n-k} \mathrm{~d} x \\
& =\sum_{k=0}^{n-1} \beta_{k} c_{n, n-k}, \quad n=\overline{1, L-1 .} .
\end{aligned}
$$

3.2.2. Computation of $p_{n}(x)$ for $n=\overline{L, H-1}$. Having found expressions for $p_{n}(x), n=\overline{1, L-1}$, we turn to derivation of equations for $p_{n}(x), n=\overline{L, H-1}$. Differential equations for $p_{n}(x)$, as in the previous case, using substitution

$$
p_{n}(x)=[1-B(x)] r_{n}(x), \quad n=\overline{L, H-1},
$$

are reduced to the form

$r_{n}^{\prime}(x)=-\lambda r_{n}(x)+\lambda \sum_{m=1}^{n-1} \omega_{n-m} r_{m}(x), \quad n=\overline{L, H-1}$

One can verify that its solution is

$$
r_{n}(x)=e^{-\lambda x} \sum_{k=0}^{n-1} \frac{(\lambda x)^{k}}{k !} c_{n, n-k}, \quad n=\overline{L, H-1}
$$

with the following recurrence relations for determination of coefficients $c_{n, m}, n=\overline{L, H-1}, m=\overline{1, n-1}$ :

$$
c_{n, n-m}=\sum_{k=m}^{n-1} \omega_{n-k} c_{k, k-m+1} .
$$

The boundary condition for determination of the only unknown coefficient $c_{n, n}$ is again obtained using the elimination method. Due to the lack of space we do not dwell on its derivation and state its final form:

$$
\begin{aligned}
& c_{n, n} \\
& =r_{n}(0)=p_{n}(0) \\
& =\lambda \omega_{n} p_{0}+\lambda \sum_{m=n+1}^{H-1}\left(\omega_{m} \alpha_{m}(0) p_{0}\right. \\
& \left.\quad+\sum_{i=1}^{n} \int_{0}^{\infty} \omega_{m-i} \alpha_{m}(x) p_{i}(x) \mathrm{d} x\right) \prod_{j=n+1}^{m-1} \alpha_{j}(0)
\end{aligned}
$$




$$
\begin{aligned}
= & \lambda \omega_{n} p_{0}+\lambda \sum_{m=n+1}^{H-1}\left(\omega_{m} \alpha_{m}(0) p_{0}\right. \\
& \left.+\sum_{i=1}^{n} \omega_{m-i} \int_{0}^{\infty} q_{m}(x) r_{i}(x) \mathrm{d} x\right) \prod_{j=n+1}^{m-1} \alpha_{j}(0) \\
= & \lambda \omega_{n} p_{0}+\lambda \sum_{m=n+1}^{H-1}\left(\omega_{m} \alpha_{m}(0) p_{0}\right. \\
& \left.+\sum_{i=1}^{n-1} \omega_{m-i} \int_{0}^{\infty} q_{m}(x) r_{i}(x) d x\right) \prod_{j=n+1}^{m-1} \alpha_{j}(0) \\
& +\lambda \sum_{m=n+1}^{H-1} \omega_{m-n} \sum_{k=0}^{n-1} \alpha_{m, k} c_{n, n-k} \prod_{j=n+1}^{m-1} \alpha_{j}(0),
\end{aligned}
$$

where for $m=\overline{n+1, H-1}, k=\overline{0, n-1}$

$$
\alpha_{m, k}=\int_{0}^{\infty} \frac{(\lambda x)^{k}}{k !} e^{-\lambda x} q_{m}(x) \mathrm{d} x .
$$

Solution of the latter equation leads to the following expression for $c_{n, n}$ :

$$
\begin{aligned}
& =\left(1-\lambda \sum_{m=n+1}^{c_{n, n}} \omega_{m-n} \alpha_{m, 0} \prod_{j=n+1}^{m-1} \alpha_{j}(0)\right)^{-1} \\
& \times\left[\lambda \omega_{n} p_{0}+\lambda \sum_{m=n+1}^{H-1} \omega_{m-n} \sum_{k=1}^{n-1} \alpha_{m, k} c_{n, n-k} \prod_{j=n+1}^{m-1} \alpha_{j}(0)\right. \\
& \quad+\lambda \sum_{m=n+1}^{H-1}\left(\omega_{m} \alpha_{m}(0) p_{0}+\sum_{i=1}^{n-1} \omega_{m-i} \int_{0}^{\infty} q_{m}(x) r_{i}(x) \mathrm{d} x\right) \\
& \left.\quad \times \prod_{j=n+1}^{m-1} \alpha_{j}(0)\right], \quad n=\overline{L, H-1} .
\end{aligned}
$$

Thus we obtain the expression for the probability that the number of customers in the system is $n, n=\overline{L, H-1}$, and it is in normal mode:

$$
p_{n}=\sum_{k=0}^{n-1} \beta_{k} c_{n, n-k}, \quad n=\overline{L, H-1} .
$$

3.2.3. Computation of $\tilde{p}_{n}(x)$ for $n=\overline{L, H-1}$. Let us dwell on the derivation of equations for $\tilde{p}_{n}(x)$, $n=\overline{L, H-1}$. Using the same approach as above, i.e., introducing new functions $\tilde{r}_{n}(x)$ defined by the relation

$$
\tilde{p}_{n}(x)=[1-B(x)] \tilde{r}_{n}(x), \quad n=\overline{L, H-1},
$$

and using this substitution in differential equations for $\tilde{p}_{n}(x)$, we arrive at the following equation for $n=$
$\overline{L, H-1}$

$$
\tilde{r}_{n}^{\prime}(x)=-\lambda_{1} \tilde{r}_{n}(x)+\lambda_{1} \sum_{m=L}^{n-1} \tilde{r}_{m}(x) \omega_{1, n-m} .
$$

It is straightforward to validate that the solution of the above equation is

$$
\tilde{r}_{n}(x)=e^{-\lambda_{1} x} \sum_{k=0}^{n-L} \frac{\left(\lambda_{1} x\right)^{k}}{k !} \tilde{c}_{n, n-k}, \quad n=\overline{L, H-1},
$$

where the coefficients $\tilde{c}_{n, m}$ are determined from the following recurrence relationships for $n=\overline{L+1, H-1}$, $m=\overline{1, n-L}$ :

$$
\tilde{c}_{n, n-m}=\sum_{k=m+L-1}^{n-1} \omega_{1, n-k} \tilde{c}_{k, k-m+1} .
$$

For the only unknown term $\tilde{c}_{n, n}$ in (27) we have the boundary condition

$$
\begin{aligned}
& \tilde{c}_{n, n} \\
& =\tilde{r}_{n}(0)=\tilde{p}_{n}(0) \\
& =\lambda \sum_{m=0}^{H-1} p_{m} \Omega_{H-m}+\lambda_{1} \sum_{m=L}^{n} \tilde{p}_{m} \Omega_{1, n+1-m} \\
& =\lambda \sum_{m=0}^{H-1} \Omega_{H-m} p_{m}+\lambda_{1} \sum_{m=L}^{n-1} \Omega_{1, n+1-m} \tilde{p}_{m} \\
& \quad+\lambda_{1} \int_{0}^{\infty} \tilde{p}_{n}(x) \mathrm{d} x \\
& =\lambda \sum_{m=0}^{H-1} \Omega_{H-m} p_{m}+\lambda_{1} \sum_{m=L}^{n-1} \Omega_{1, n+1-m} \tilde{p}_{m} \\
& \quad+\lambda_{1} \sum_{k=0}^{n-L} \tilde{\beta}_{k} \tilde{c}_{n, n-k}, \quad n=\overline{L, H-1},
\end{aligned}
$$

where

$$
\begin{aligned}
\tilde{\beta}_{k} & =\frac{\lambda_{1}^{k}}{k !} \int_{0}^{\infty} x^{k} e^{-\lambda_{1} x}[1-B(x)] \mathrm{d} x \\
& =\frac{\left(-\lambda_{1}\right)^{k}}{k !} \tilde{\beta}^{(k)}\left(\lambda_{1}\right) .
\end{aligned}
$$

Solution of the above equation gives the expression for $\tilde{c}_{n, n}, n=\overline{L, H-1}$ :

$$
\begin{aligned}
& \tilde{c}_{n, n} \\
& =\left(1-\lambda_{1} \tilde{\beta}_{0}\right)^{-1}\left(\lambda \sum_{m=0}^{H-1} \Omega_{H-m} p_{m}\right. \\
& \left.\quad+\lambda_{1} \sum_{m=L}^{n-1} \Omega_{1, n+1-m} \tilde{p}_{m}+\lambda_{1} \sum_{k=1}^{n-L} \tilde{\beta}_{k} \tilde{c}_{n, n-k}\right) .
\end{aligned}
$$


Thus we have obtained the expression for the probability that the number of customers in the system is $n, n=$ $\overline{L, H-1}$, and it is in overload mode:

$$
\begin{aligned}
\tilde{p}_{n} & =\int_{0}^{\infty}[1-B(x)] e^{-\lambda_{1} x} \sum_{k=0}^{n-L} \frac{\left(\lambda_{1} x\right)^{k}}{k !} \tilde{c}_{n, n-k} \mathrm{~d} x \\
& =\sum_{k=0}^{n-L} \tilde{\beta}_{k} \tilde{c}_{n, n-k}, \quad n=\overline{L, H-1} .
\end{aligned}
$$

3.2.4. Computation of $\boldsymbol{p}_{\boldsymbol{H}}(\boldsymbol{x})$. The differential equation for $p_{H}(x)$ using substitution

$$
p_{H}(x)=[1-B(x)] r_{H}(x)
$$

can be reduced to

$$
\begin{aligned}
r_{H}^{\prime}(x)= & -\lambda_{1} r_{H}(x)+\lambda \sum_{m=1}^{H-1} \omega_{H-m} r_{m}(x) \\
& +\lambda_{1} \sum_{m=L}^{H-1} \omega_{1, H-m} \tilde{r}_{m}(x) .
\end{aligned}
$$

Its solution can be written in the form

$$
\begin{aligned}
& r_{H}(x) \\
& =e^{-\lambda_{1} x}\left(c_{H, H}^{*}+\lambda \int_{0}^{x} e^{\lambda_{1} y} \sum_{m=1}^{H-1} \omega_{H-m} r_{m}(y) \mathrm{d} y\right. \\
& \left.+\lambda_{1} \int_{0}^{x} e^{\lambda_{1} y} \sum_{m=L}^{H-1} \omega_{1, H-m} \tilde{r}_{m}(y) \mathrm{d} y\right) \\
& =e^{-\lambda x} \sum_{k=0}^{H-2} \frac{\left(\lambda_{2} x\right)^{k}}{k !} c_{H, H-k}+e^{-\lambda_{1} x}\left[c_{H, H}^{*}\right. \\
& +\sum_{k=0}^{H-2} \sum_{m=k+1}^{H-1} \omega_{H-m}\left(\frac{\lambda}{\lambda_{2}}\right)^{k+1} c_{m, m-k} \\
& \left.+\sum_{k=1}^{H-L} \frac{\left(\lambda_{1} x\right)^{k}}{k !} \sum_{m=L-k+1}^{H-1} \omega_{1, H-m} \tilde{c}_{m, m-k+1}\right] \text {. }
\end{aligned}
$$

Thus the final expression for $r_{H}(x)$ can be represented as two finite sums, i.e.,

$$
\begin{aligned}
r_{H}(x)= & e^{-\lambda x} \sum_{k=0}^{H-2} \frac{\left(\lambda_{2} x\right)^{k}}{k !} c_{H, H-k} \\
& +e^{-\lambda_{1} x} \sum_{k=0}^{H-L} \frac{\left(\lambda_{1} x\right)^{k}}{k !} \tilde{c}_{H, H-k},
\end{aligned}
$$

where

$$
\begin{array}{r}
c_{H, H-k}=-\sum_{i=k}^{H-2} \sum_{m=i+1}^{H-1} \omega_{H-m}\left(\frac{\lambda}{\lambda_{2}}\right)^{i+1} c_{m, m-k}, \\
k=\overline{0, H-2},
\end{array}
$$

$$
\begin{aligned}
\tilde{c}_{H, H} & =c_{H, H}^{*} \\
& +\sum_{k=0}^{H-2} \sum_{m=k+1}^{H-1} \omega_{H-m}\left(\frac{\lambda}{\lambda_{2}}\right)^{k+1} c_{m, m-k}, \\
\tilde{c}_{H, H-k} & =\sum_{m=L+k-1}^{H-1} \omega_{1, H-m} \tilde{c}_{m, m-k+1}, \\
k & =\overline{1, H-L} .
\end{aligned}
$$

The boundary condition, which is determined using the elimination method as well, has the form

$$
\begin{aligned}
& c_{H, H}+\tilde{c}_{H, H} \\
& =r_{H}(0)=p_{H}(0) \\
& =\lambda \sum_{m=0}^{H-1} p_{m} \Omega_{H+1-m}+\lambda p_{0} \omega_{H} \\
& \quad+\lambda_{1} \sum_{m=L}^{H-1} \tilde{p}_{m} \Omega_{1, H+1-m}+\lambda_{1} \int_{0}^{\infty} p_{H}(x) \mathrm{d} x \\
& =\lambda p_{0} \omega_{H}+\sum_{m=0}^{H-1} \lambda p_{m} \Omega_{H+1-m}+\lambda_{1}\left[\sum_{m=L}^{H-1} \tilde{p}_{m} \Omega_{1, H+1-m}\right. \\
& \left.\quad+\sum_{k=0}^{H-2}\left(\frac{\lambda_{2}}{\lambda}\right)^{k} \beta_{k} c_{H, H-k}+\sum_{k=0}^{H-L} \tilde{\beta}_{k} \tilde{c}_{H, H-k}\right] .
\end{aligned}
$$

After collecting the common terms, one can find the following expression for $\tilde{c}_{H, H}$ :

$$
\begin{aligned}
& \tilde{c}_{H, H} \\
& =\left(1-\lambda_{1} \tilde{\beta}_{0}\right)^{-1}\left(\lambda p_{0} \omega_{H}+\sum_{m=0}^{H-1} \lambda p_{m} \Omega_{H+1-m}\right. \\
& \quad+\lambda_{1}\left[\sum_{m=L}^{H-1} \tilde{p}_{m} \Omega_{1, H+1-m}+\sum_{k=0}^{H-2}\left(\frac{\lambda_{2}}{\lambda}\right)^{k} \beta_{k} c_{H, H-k}\right. \\
& \left.\left.\quad+\sum_{k=1}^{H-L} \tilde{\beta}_{k} \tilde{c}_{H, H-k}\right]-c_{H, H}\right) .
\end{aligned}
$$

Note that the probability that the number of customers in 
the system is $H$ and the system is in overload mode equals

$$
\begin{aligned}
p_{H}= & \int_{0}^{\infty}[1-B(x)]\left(e^{-\lambda x} \sum_{k=0}^{H-2} \frac{\left(\lambda_{2} x\right)^{k}}{k !} c_{H, H-k}\right. \\
& \left.+e^{-\lambda_{1} x} \sum_{k=0}^{H-L} \frac{\left(\lambda_{1} x\right)^{k}}{k !} \tilde{c}_{H, H-k}\right) \mathrm{d} x \\
= & \sum_{k=0}^{H-2}\left(\frac{\lambda_{2}}{\lambda}\right)^{k} \beta_{k} c_{H, H-k}+\sum_{k=0}^{H-L} \tilde{\beta}_{k} \tilde{c}_{H, H-k} .
\end{aligned}
$$

3.2.5. Computation of functions $p_{\boldsymbol{n}}(x)$ for $\boldsymbol{n}=\overline{\boldsymbol{H}+\mathbf{1}, \boldsymbol{R}-\mathbf{1}}$. Whereas probabilities $p_{n}(x)$, $n=\overline{1, H}$, and $\tilde{p}_{n}(x), n=\overline{L, H-1}$, are now considered to be found, we turn to the determination of probabilities $p_{n}(x), \quad n=\overline{H+1, R-1}$. We seek to obtain their expressions in the form

$$
\begin{aligned}
& p_{n}(x) \\
& =[1-B(x)]\left[e^{-\lambda x} \sum_{k=0}^{H-2} \frac{\left(\lambda_{2} x\right)^{k}}{k !} c_{n, n-k}\right. \\
& \left.+e^{-\lambda_{1} x} \sum_{k=0}^{n-L} \frac{\left(\lambda_{1} x\right)^{k}}{k !} \tilde{c}_{n, n-k}\right] \\
& n=\frac{H+1, R-1}{H+1}
\end{aligned}
$$

The differential equation for $p_{n}(x)$ using substitution

$$
p_{n}(x)=[1-B(x)] r_{n}(x), \quad n=\overline{H+1, R-1},
$$

is reduced to

$$
\begin{aligned}
& r_{n}^{\prime}(x) \\
& =-\lambda_{1} r_{n}(x)+\lambda \sum_{m=1}^{H-1} \omega_{n-m} r_{m}(x) \\
& \quad+\lambda_{1} \sum_{m=L}^{H-1} \omega_{1, n-m} \tilde{r}_{m}(x)+\lambda_{1} \sum_{m=H}^{n-1} \omega_{1, n-m} r_{m}(x) .
\end{aligned}
$$

The solution of the latter equation is given by

$$
\begin{aligned}
& r_{n}(x) \\
& =e^{-\lambda x} \sum_{k=0}^{H-2} \frac{\left(\lambda_{2} x\right)^{k}}{k !} c_{n, n-k} \\
& +e^{-\lambda_{1} x} \sum_{k=0}^{n-L} \frac{\left(\lambda_{1} x\right)^{k}}{k !} \tilde{c}_{n, n-k}, \quad n=\overline{H+1, R-1}
\end{aligned}
$$

where

$$
\begin{aligned}
& c_{n, n-k}=-\left[\frac{\lambda_{1}}{\lambda_{2}} \sum_{m=H}^{n-1} \sum_{i=k}^{H-2} \omega_{1, n-m} c_{m, m-i}\right. \\
&\left.+\sum_{i=k}^{H-2}\left(\frac{\lambda}{\lambda_{2}}\right)^{i+1} \sum_{m=i+1}^{H-1} \omega_{n-m} c_{m, m-i}\right], \\
& n=\overline{H+1, R-1}, \quad k=\overline{0, H-2}, \\
& \tilde{c}_{n, n}= \sum_{k=0}^{H-2}\left(\frac{\lambda}{\lambda_{2}}\right)^{k+1} \sum_{m=k+1}^{H-1} \omega_{n-m} c_{m, m-k} \\
&+\frac{\lambda_{1}}{\lambda_{2}} \sum_{k=0}^{H-2} \sum_{m=H}^{n-1} \omega_{1, n-m} c_{m, m-k}+c_{n, n}^{*}, \\
& n=\overline{H+1, R-1}
\end{aligned}
$$

$$
\begin{aligned}
\tilde{c}_{n, n-k}= & \sum_{m=L+k-1}^{H-1} \omega_{1, n-m} \tilde{c}_{m, m-k+1} \\
& +\sum_{m=H}^{n-1} \omega_{1, n-m} \tilde{c}_{m, m-k+1}, \\
\tilde{c}_{n, n-k}= & \sum_{m=L+k-1}^{n-1} \omega_{1, n-m} \tilde{c}_{m, m-k+1}, \\
& \quad n=\overline{H+1, R-1}, \quad k=\overline{1, H-L}, \quad k=\overline{H-L+1, n-L} .
\end{aligned}
$$

The only term left to de determined is $\tilde{c}_{n, n}$. It is found from boundary conditions obtained using the elimination method, whose derivation here is omitted due to the lack of space. We have, for $n=\overline{H+1, R-1}$,

$$
\begin{aligned}
& c_{n, n}+\tilde{c}_{n, n} \\
& =r_{n}(0)=p_{n}(0) \\
& =\lambda p_{0} \sum_{m=n}^{R-1} \omega_{m} \prod_{j=n+1}^{m} \alpha_{j}(0) \\
& \quad+\lambda_{1} \sum_{m=n+1}^{R-1} \omega_{1, m-n}\left[\sum_{k=0}^{H-2}\left(\frac{\lambda_{2}}{\lambda}\right)^{k} \alpha_{m, k} c_{n, n-k}\right. \\
& \left.\quad+\sum_{k=0}^{n-L} \tilde{\alpha}_{m, k} \tilde{c}_{n, n-k}\right] \prod_{j=n+1}^{m-1} \alpha_{j}(0) \\
& \quad+\sum_{m=n+1}^{R-1} \int_{0}^{\infty}\left(\lambda \sum_{i=1}^{H-1} r_{i}(x) \omega_{m-i}+\lambda_{1} \sum_{i=L}^{H-1} \tilde{r}_{i}(x) \omega_{1, m-i}\right. \\
& \left.\quad+\lambda_{1} \sum_{i=H}^{n-1} r_{i}(x) \omega_{1, m-i}\right) q_{m}(x) \mathrm{d} x \prod_{j=n+1}^{m-1} \alpha_{j}(0),
\end{aligned}
$$


where for $\alpha_{m, k}$ and $\tilde{\alpha}_{m, k}$ the following notation is used:

$$
\begin{aligned}
\alpha_{m, k}= & \int_{0}^{\infty} \frac{(\lambda x)^{k}}{k !} e^{-\lambda x} q_{m}(x) \mathrm{d} x, \\
& m=\overline{n+1, R-1}, \quad k=\overline{0, H-2}, \\
\tilde{\alpha}_{m, k}= & \int_{0}^{\infty} \frac{\left(\lambda_{1} x\right)^{k}}{k !} e^{-\lambda_{1} x} q_{m}(x) \mathrm{d} x, \\
& m=\overline{n+1, R-1}, \quad k=\overline{0, n-L} .
\end{aligned}
$$

Collecting the common terms in the previous equation, we get

$$
\begin{aligned}
\tilde{c}_{n, n} & \left(1-\lambda_{1} \sum_{m=n+1}^{R-1} \omega_{1, m-n} \tilde{\alpha}_{m, 0} \prod_{j=n+1}^{m-1} \alpha_{j}(0)\right)^{-1} \\
& \times\left[\lambda p_{0} \sum_{m=n}^{R-1} \omega_{m} \prod_{j=n+1}^{m} \alpha_{j}(0)\right. \\
+ & \lambda_{1} \sum_{m=n+1}^{R-1} \omega_{1, m-n}\left(\sum_{k=0}^{H-2}\left(\frac{\lambda_{2}}{\lambda}\right)^{k} \alpha_{m, k} c_{n, n-k}\right. \\
+ & \sum_{k=1}^{n-L} \tilde{\alpha}_{m, k} \tilde{c}_{n, n-k} \prod_{j=n+1}^{m-1} \alpha_{j}(0) \\
+ & \sum_{m=n+1}^{R-1} \int_{0}^{\infty}\left(\lambda \sum_{i=1}^{H-1} r_{i}(x) \omega_{m-i}+\lambda_{1} \sum_{i=L}^{H-1} \tilde{r}_{i}(x) \omega_{1, m-i}\right. \\
& \left.\left.+\lambda_{1} \sum_{i=H}^{n-1} r_{i}(x) \omega_{1, m-i}\right) q_{m}(x) \mathrm{d} x \prod_{j=n+1}^{m-1} \alpha_{j}(0)-c_{n, n}\right],
\end{aligned}
$$

Now we can find the probability that the number of customers in the system is $n, n=\overline{H+1, R-1}$, and it is in overload mode. We have

$$
\begin{aligned}
& p_{n} \\
& =\int_{0}^{\infty}[1-B(x)]\left(e^{-\lambda x} \sum_{k=0}^{H-2} \frac{\left(\lambda_{2} x\right)^{k}}{k !} c_{n, n-k}\right. \\
& \left.\quad+e^{-\lambda_{1} x} \sum_{k=0}^{n-L} \frac{\left(\lambda_{1} x\right)^{k}}{k !} \tilde{c}_{n, n-k}\right) \mathrm{d} x \\
& =\sum_{k=0}^{H-2}\left(\frac{\lambda_{2}}{\lambda}\right)^{k} \beta_{k} c_{n, n-k}+\sum_{k=0}^{n-L} \tilde{\beta}_{k} \tilde{c}_{n, n-k}, \\
& \quad n=\overline{H+1, R-1 .}
\end{aligned}
$$

3.2.6. Computation of $\tilde{\boldsymbol{p}}_{\boldsymbol{R}}(\boldsymbol{x})$. Let us now find the expression for the probability $\tilde{p}_{R}(x)$, in the form

$$
\begin{aligned}
\tilde{p}_{R}(x)= & {[1-B(x)]\left[c^{*}+e^{-\lambda x} \sum_{k=0}^{H-2} \frac{(\lambda x)^{k}}{k !} c_{R, R-k}\right.} \\
& +e^{-\lambda_{1} x} \sum_{k=0}^{R-L-1} \frac{\left(\lambda_{1} x\right)^{k}}{k !} \tilde{c}_{R, R-k] .}
\end{aligned}
$$

Having introduced function $\tilde{p}_{R}(x)$ by the relation

$$
\tilde{p}_{R}(x)=[1-B(x)] \tilde{r}_{R}(x),
$$

we obtain from the differential equation for $\tilde{p}_{R}(x)$ the corresponding equation for $\tilde{r}_{R}(x)$. We have

$$
\begin{aligned}
\tilde{r}_{R}^{\prime}(x)= & \lambda \sum_{m=1}^{H-1} \Omega_{R-m} r_{m}(x) \\
& +\lambda_{1} \sum_{m=L}^{H-1} \Omega_{1, R-m} \tilde{r}_{m}(x) \\
& +\lambda_{1} \sum_{m=H}^{R-1} \Omega_{1, R-m} r_{m}(x) .
\end{aligned}
$$

The solution of the latter equation is

$$
\begin{aligned}
\tilde{r}_{R}(x)= & c^{*}+e^{-\lambda x} \sum_{i=0}^{H-2} \frac{(\lambda x)^{i}}{i !} c_{R, R-i} \\
& +e^{-\lambda_{1} x} \sum_{i=0}^{R-1-L} \frac{\left(\lambda_{1} x\right)^{i}}{i !} \tilde{c}_{R, R-i}
\end{aligned}
$$

where

$$
\begin{aligned}
c^{*}= & c_{R, R}^{*}+\sum_{k=0}^{H-2} \sum_{m=k+1}^{H-1} \Omega_{R-m} c_{m, m-k} \\
& +\sum_{k=0}^{R-1-L} \sum_{m=L+k}^{R-1} \Omega_{1, R-m} \tilde{c}_{m, m-k} \\
& +\sum_{k=0}^{H-2} \sum_{m=H}^{R-1} \frac{\lambda_{1} \lambda_{2}^{k}}{\lambda^{k+1}} \Omega_{1, R-m} c_{m, m-k},
\end{aligned}
$$

$$
\begin{aligned}
c_{R, R-i}= & -\sum_{k=i}^{H-2} \sum_{m=k+1}^{H-1} \Omega_{R-m} c_{m, m-k} \\
& -\sum_{k=i}^{H-2} \sum_{m=H}^{R-1} \frac{\lambda_{1} \lambda_{2}^{k}}{\lambda^{k+1}} \Omega_{1, R-m} c_{m, m-k}, \\
i=\overline{0, H-2}, & \sum_{k=i}^{R-1-L} \sum_{m=L+k}^{R-1} \Omega_{1, R-m} \tilde{c}_{m, m-k},
\end{aligned}
$$




$$
i=\overline{0, R-1-L} \text {. }
$$

From the boundary condition we find that for unknown term $c^{*}$ in 43 it holds that

$$
c_{R, R}+\tilde{c}_{R, R}+c^{*}=\tilde{r}_{R}(0)=\tilde{p}_{R}(0)=\lambda p_{0} \Omega_{R} .
$$

Hence

$$
c^{*}=\lambda p_{0} \Omega_{R}-c_{R, R}-\tilde{c}_{R, R} .
$$

The probability that the number of customers in the system in $R$ (and it is in discard mode) is given by

$$
p_{R}=b c^{*}+\sum_{k=0}^{H-2} \beta_{k} c_{R, R-k}+\sum_{k=0}^{R-L-1} \tilde{\beta}_{k} \tilde{c}_{R, R-k} .
$$

3.2.7. Computation of the functions $\tilde{p}_{n}(x)$ for $\boldsymbol{n}=\overline{\boldsymbol{H}+\mathbf{1}, \boldsymbol{R}-\mathbf{1}}$. The last expressions that are left to be found are those for probabilities $\tilde{p}_{n}(x), \quad n=$ $\overline{H+1, R-1}$. Write

$$
\begin{aligned}
\alpha^{*}= & \int_{0}^{\infty} \tilde{p}_{R}(x) \frac{b(x)}{1-B(x)} \mathrm{d} x \\
= & \int_{0}^{\infty} \tilde{r}_{R}(x) b(x) \mathrm{d} x \\
= & \sum_{k=0}^{H-2} \frac{(-\lambda)^{k}}{k !} \beta^{(k)}(\lambda) c_{R, R-k} \\
& +\sum_{k=0}^{R-L} \frac{\left(-\lambda_{1}\right)^{k}}{k !} \beta^{(k)}\left(\lambda_{1}\right) \tilde{c}_{R, R-k} .
\end{aligned}
$$

One can readily verify that differential equations for $\tilde{p}_{n}(x)$ using substitution

$$
\tilde{p}_{n}(x)=[1-B(x)] \tilde{r}_{n}(x), \quad n=\overline{H+1, R-1},
$$

can be reduced to

$$
\tilde{r}_{n}^{\prime}(x)=0, \quad n=\overline{H+1, R-1},
$$

whose solution is

$$
\tilde{r}_{n}(x)=\tilde{c}_{n}, \quad n=\overline{H+1, R-1} .
$$

The boundary condition used to obtain the expression for unknown term $\tilde{c}_{n}$ in this case is

$$
\begin{aligned}
\tilde{c}_{n} & =\tilde{r}_{n}(0)=\tilde{p}_{n}(0) \\
& =\int_{0}^{\infty} \tilde{p}_{n+1}(x) \frac{b(x)}{1-B(x)} \mathrm{d} x \\
& =\int_{0}^{\infty} \tilde{r}_{n+1}(x) b(x) \mathrm{d} x \\
& =\tilde{c}_{n+1}=\alpha^{*}, \quad n=\overline{H+1, R-1} .
\end{aligned}
$$

Thus for $\tilde{p}_{n}(x), n=\overline{H+1, R-1}$, we have

$$
\tilde{p}_{n}(x)=[1-B(x)] \tilde{r}_{n}(x), \quad n=\overline{H+1, R-1},
$$

and integration from 0 to $\infty$ yields the expression for $\tilde{p}_{n}$ :

$$
\begin{aligned}
\tilde{p}_{n} & =\int_{0}^{\infty} \tilde{p}_{n}(x) \mathrm{d} x \\
& =\int_{0}^{\infty}[1-B(x)] \tilde{r}_{n}(x) \mathrm{d} x \\
& =\tilde{c}_{n} b=\alpha^{*} b, \quad n=\overline{H+1, R-1} .
\end{aligned}
$$

3.2.8. Computation of $\boldsymbol{p}_{0}$. The stationary probability $p_{0}$ of an empty system is determined, as usual, from the normalization condition:

$$
p_{0}+\sum_{n=1}^{R-1} p_{n}+\sum_{n=L}^{H-1} \tilde{p}_{n}+\sum_{n=H+1}^{R} \tilde{p}_{n}=1 .
$$

Now we state the main result of Section 3.

Theorem 1. The stationary probability density $p_{n}(x)$ that the total number of customers in the system is $n$, $n=\overline{1, L-1}$, the elapsed service time of a currently served customer is $x$ and the system is in normal mode is given by the relation 10 . All quantities in this relation are given by (13), (16) and (17).

The stationary probability density $p_{n}(x)$ that the total number of customers in the system is $n, n=\overline{L, H-1}$, the elapsed service time of a currently served customer is $x$ and the system is in normal mode is given by the relation (19). All quantities in this relation are given by (20), (21), (22) and 23.

The stationary probability density $\tilde{p}_{n}(x)$ that the total number of customers in the system is $n, n=\overline{L, H-1}$, the elapsed service time of a currently served customer is $x$ and the system is in overload mode is given by the relation (25). All quantities in this relation are given by (26), (27), 28) and (29).

The stationary probability density $p_{H}(x)$ that the total number of customers in the system is $H$, the elapsed service time of a currently served customer is $x$ and the system is in overload mode is given by the relation (31). All quantities in this relation are given by (32), (33) and (34).

The stationary probability density $p_{n}(x)$ that the total number of customers in the system is $n, n=\overline{H, R-1}$, the elapsed service time of a currently served customer is $x$ and the system is in overload mode is given by the relation (36). All quantities in this relation are given by (37), (38), (39) and (40).

The stationary probability density $\tilde{p}_{R}(x)$ that the total number of customers in the system is $R$, the elapsed 
service time of a currently served customer is $x$ and the system is in discard mode is given by the relation (42). All quantities in this relation are given by (43), (44), (45) and (46).

The stationary probability density $\tilde{p}_{n}(x)$ that the total number of customers in the system is $n$, $n=\overline{H+1, R-1}$, the elapsed service time of a currently served customer is $x$ and the system is in discard mode is given by the relation (49). The quantity $\alpha^{*}$ in this relation is given by 48).

The stationary probabilities $p_{n}$ and $\tilde{p}_{n}$ of $n$ customers in the system (and the system's corresponding state) are given by (18), (24), (30), (35), (41), (47) and (50).

The stationary probability $p_{0}$ of an empty system is determined from the normalization condition (51).

3.3. Performance characteristics. The stationary probability $P\left(S_{1}\right)$ of the system being in overload mode is equal to the sum of the corresponding probabilities, i.e.,

$$
P\left(S_{1}\right)=\sum_{n=H}^{R-1} p_{n}+\sum_{n=L}^{H-1} \tilde{p}_{n} .
$$

Similarly, we can find the stationary probability $P\left(S_{2}\right)$ of the system being in discard mode. We have

$$
P\left(S_{2}\right)=\sum_{n=H+1}^{R} \tilde{p}_{n}
$$

Finally the stationary probability $P\left(S_{0}\right)$ of the system being in normal mode is

$$
P\left(S_{0}\right)=\sum_{n=0}^{H-1} p_{n}
$$

In order to find the mean time $\tau_{12}$ the system spends in overload and the discard set of states during one control cycle, one needs to find at first $\tau$, by which we denote the mean control cycle length. A control cycle is the time between consecutive transitions from overload state $L$ to normal state $(L-1)$. The rate of such transitions in steady state equals

$$
\mu=\int_{0}^{\infty} \frac{b(x)}{1-B(x)} \tilde{p}_{L}(x) \mathrm{d} x=\tilde{c}_{L, L} \beta\left(\lambda_{1}\right) .
$$

Using renewal theory, according to which the mean cycle length is the reciprocal of the transition rate in steady state, we get $\tau=1 / \mu$.

The control cycle consists of two parts: the time system spends in normal mode and the time the system spends in overload and discard modes. Respective probabilities are equal to $P\left(S_{1}\right)+P\left(S_{2}\right)$ and $P\left(S_{0}\right)$.
Thus, the mean time $\tau_{12}$ can be calculated by the following formula:

$$
\tau_{12}=\frac{P\left(S_{1}\right)+P\left(S_{2}\right)}{P\left(S_{0}\right)+P\left(S_{1}\right)+P\left(S_{2}\right)} \tau .
$$

\section{Policy P2}

In this section we turn to the $M^{[X]}|G| 1\langle L, H\rangle \mid\langle H, R\rangle$ system operating under policy $\mathrm{P} 2$. As mentioned earlier, according to policy $\mathrm{P} 2$, the system may switch between operating modes only at the time instant of customer departure. If in normal mode, just before the customer departure, the total number of customers in the system equals $H$, then the system switches to overload mode. Similarly, if in overload mode just before the customer departure the total number of customers in the system equals $H$, then the system switches to discard mode. Suppose the batch size distributions are the same, hence the probability that the arriving batch of any type contains exactly $n, n \geq 1$, customers equals $\omega_{n}$. For uniformity, in this section we use the notation $\lambda_{0}$ for the total intensity of arrivals, i.e., $\lambda_{0}=\lambda_{1}+\lambda_{2}$.

Here we develop a Markov chain embedded at customer departure epochs and get its transition probability matrix for computing the stationary distribution of the Markov chain. After that we get the stationary queue length distribution of the corresponding Markov process. Finally, we find the stationary probabilities of the system being in each of operating modes, the stationary expectation control cycle time and the stationary mean return time from overload and discard modes to normal mode.

4.1. Embedded Markov chain. Let us denote by $\{X(t), t \geq 0\}$ a two-dimensional stochastic process with the set of states

$$
S=\left\{\begin{array}{l|l}
(j, s) & \begin{array}{l}
j=\overline{0, R}, \\
j=\overline{L, R}, \\
j=\overline{H+1, R-1},
\end{array}=2
\end{array}\right\}
$$

and its subsets $S_{i}=\{(j, s) \in S \mid s=i\}, i=\{0,1,2\}$, where $j$ is the number of customers in the system at the instant $t \geq 0$ and $s$ indicates system operating mode.

Take the service completion epochs to be $0<t_{1}<$ $t_{2}<\ldots$, where $t_{n}$ is the departure instant of the $n$-th customer. According to policy P2, we assume that the system operating mode $s$ may be changed only at the completion of a service $t_{n}$. Then the discrete-time process embedded at customer departure epochs $X\left(t_{n}+\right.$ $0)$ emerges as a Markov chain.

The set of states of $X\left(t_{n}+0\right)$ and its subsets are as 
follows:

$$
\begin{gathered}
\tilde{S}=\left\{(j, s) \mid \begin{array}{ll}
j=\overline{0, H-2}, & s=0 \\
j=\overline{L, R-2}, & s=1 \\
j=\overline{H+1, R-1}, & s=2
\end{array}\right\}, \\
\tilde{S}_{i}=\{(j, s) \in \tilde{S} \mid s=i\}, \quad i=\{0,1,2\} .
\end{gathered}
$$

Let us denote by $\left\{p_{j, s}\right\}$ and $\left\{q_{j, s}\right\}$ the stationary distributions of $X(t)$ and $X\left(t_{n}+0\right)$, respectively,

$$
\begin{gathered}
p_{j, s}=\lim _{t \rightarrow \infty} P\{X(t)=(j, s)\}, \quad(j, s) \in S, \\
q_{j, s}=\lim _{n \rightarrow \infty} P\left\{X\left(t_{n}+0\right)=(j, s)\right\}, \quad(j, s) \in \tilde{S} .
\end{gathered}
$$

To obtain transition probabilities of the Markov chain, we denote by $\beta_{k}^{s}, s=0,1, k \geq 0$, the probability that in operating mode $s$ during the service time of a customer exactly $k$ batches will arrive at the system:

$$
\beta_{k}^{s}=\int_{0}^{\infty} e^{-\lambda_{s} x} \frac{\left(\lambda_{s} x\right)^{k}}{k !} \mathrm{d} B(x)=\frac{\lambda_{s}^{k}}{k !} \beta^{(k)}\left(\lambda_{s}\right) .
$$

To express transition probabilities of the Markov chain, we introduce the probability $\omega_{i}^{k}, k \geq 0, i \geq 0$, that there are exactly $i$ customers in $k$ batches,

$$
\begin{aligned}
\omega_{i}^{0} & =\delta_{i}, \quad i \geq 0, \\
\omega_{i}^{k} & =\sum_{n=0}^{i} \omega_{i-n}^{k-1} \omega_{n}, \quad k \geq 1, \quad i \geq 0,
\end{aligned}
$$

where $\delta_{i}$ is the Kronecker delta $\left(\delta_{i}=1\right.$ if $i=0$, or 0 otherwise).

We introduce $\alpha_{i}^{s}, \quad s=0,1, \quad i \geq 0$, i.e., the probability that in operating mode $s$ exactly $i$ new customers arrive during the time of service of a customer, and $A_{i}^{s}, \quad s=0,1, \quad i \geq 0$, i.e., the probability that in operating mode $s$ no less than $i$ new customers arrive during the time of service of a customer. We also introduce $\gamma_{i}, i \geq 0$, i.e., the probability that immediately after the departure of the customer who arrived when the system was empty there will be exactly $i$ customers in the system, and $\Gamma_{R-1}$, i.e., the probability that immediately after the departure of the customer who arrived when the system was empty, there will be no less than $R-1$ customers in the system. Then

$$
\begin{aligned}
\alpha_{i}^{s} & =\sum_{k=0}^{i} \beta_{k}^{s} \omega_{i}^{k}, \quad A_{i}^{s}=\sum_{k=i}^{\infty} \alpha_{k}^{s}, \quad s=0,1, \quad i \geq 0, \\
\gamma_{i} & =\sum_{k=1}^{i+1} \omega_{k}^{0} \alpha_{i-k+1}^{0}, \quad i \geq 0, \quad \Gamma_{R-1}=\sum_{i=R-1}^{\infty} \gamma_{i} .
\end{aligned}
$$

Thus the equilibrium equation set for probability distribution $\left\{q_{j, s}\right\}$ takes the form

$$
\begin{aligned}
q_{j, 0}= & q_{0,0} \gamma_{j}+\sum_{i=1}^{\min (j+1, H-2)} q_{i, 0} \alpha_{j-i+1}^{0} \\
& +\delta_{j-L+1} q_{L, 1} \alpha_{0}^{1}, \quad j=\overline{0, H-2}, \\
q_{j, 1}= & q_{0,0} \gamma_{j}+\sum_{i=1}^{H-2} q_{i, 0} \alpha_{j-i+1}^{0} \\
& +\sum_{i=L}^{\min (j+1, R-2)} q_{i, 1} \alpha_{j-i+1}^{1} \\
& +\delta_{j-} q_{H+1,2}, \quad j=\overline{H-1, R-2}, \\
q_{j, 1}= & \sum_{i=L}^{j+1} q_{i, 1} \alpha_{j-i+1}^{1}, \quad j=\overline{L, H-2}, \\
q_{R-1,2}= & q_{0,0} \Gamma_{R-1} \\
& +\sum_{i=1}^{H-2} q_{i, 0} A_{R-i}^{k}+\sum_{i=L}^{R-2} q_{i, 1} A_{R-i}^{1}, \\
q_{j, 2}= & q_{R-1,2}, \quad j=\overline{H+1, R-2} .
\end{aligned}
$$

The stationary probability $q_{0,0}$ of an empty system is determined, as usual, from the normalization condition:

$$
\sum_{j=0}^{H-1} q_{j, 0}+\sum_{j=L}^{R-2} q_{j, 1}+\sum_{j=H+1}^{R-1} q_{j, 2}=1 .
$$

The equilibrium equation set (53) is of a peculiar feature, so we can use the technique based on the exclusion of certain states of the Markov chain and the bonding of the remaining subsets (Bocharov et al., 2004) for its solving.

4.2. Stationary state distribution. We use renewal theory to receive the stationary queue length distribution of the corresponding stochastic process from the stationary queue length distribution of the embedded Markov chain.

The stationary mean $T$ of the time interval between neighboring instants $t_{n}$ and $t_{n+1}$ is defined by the formula $T=b+\frac{1}{\lambda_{0}} q_{0,0}$. We also write $\nu=1 / T$, and

$$
\begin{aligned}
\tilde{\beta}_{k}^{s} & =\int_{0}^{\infty}[1-B(x)] e^{-\lambda_{s} x} \frac{\left(\lambda_{s} x\right)^{k}}{k !} \mathrm{d} x \\
& =\frac{\lambda_{s}^{k}}{k !} \tilde{\beta}^{(k)}\left(\lambda_{s}\right), \\
\tilde{\alpha}_{i}^{s} & =\sum_{k=0}^{i} \tilde{\beta}_{k}^{s} \omega_{i}^{k}, \\
\tilde{A}_{i}^{s} & =\sum_{k=i}^{\infty} \tilde{\alpha}_{k}^{s}, \quad s=0,1, \quad i \geq 0,
\end{aligned}
$$




$$
\begin{gathered}
\tilde{\gamma}_{i}=\sum_{k=1}^{i} \omega_{k}^{0} \tilde{\alpha}_{i-k}^{0},, \quad i \geq 1, \\
\tilde{\Gamma}_{R}=\sum_{i=R}^{\infty} \tilde{\gamma}_{i} .
\end{gathered}
$$

The following theorem contains formulas for calculating the stationary queue length distribution of the corresponding Markov process.

Theorem 2. The stationary probabilities of the stochastic process $X(t)$ are given by $p_{0,0}=\frac{\nu}{\lambda_{0}} q_{0,0}$,

$$
\begin{aligned}
p_{j, 0} & =\nu\left(\tilde{\gamma}_{j} q_{0,0}+\sum_{i=1}^{\min (j, H-2)} \tilde{\alpha}_{j-i}^{0} q_{i, 0}\right), \quad j=\overline{1, R-1}, \\
p_{R, 0} & =\nu\left(\tilde{\Gamma}_{R} q_{0,0}+\sum_{i=1}^{H-2} \tilde{A}_{j-i}^{0} q_{i, 0}\right), \\
p_{j, 1} & =\nu \sum_{i=L}^{\min (j, R-2)} \tilde{\alpha}_{j-i}^{1} q_{i, 1}, \quad j=\overline{L . R-1}, \\
p_{R, 1} & =\nu \sum_{i=L}^{R-2} \tilde{A}_{j-i}^{1} q_{i, 1}, \\
p_{j, 2} & =\nu b q_{R-1,2}, \quad j=\overline{H+1, R-1},
\end{aligned}
$$

where $q_{i, s}$ are the solutions of the system (53) with the normalization condition (54).

Using renewal theory one can obtain the other stationary probabilistic characteristics, for example, a stationary density $p_{j, s}(x)$, i.e., the probability that in operating mode $s$ exactly $j$ customers are in the system and the time interval after the last customer is departure is $x$.

4.3. Performance characteristics. Now we give the same performance characteristics as were defined for the system operating under policy P1 in Section 3.

The stationary probability $P\left(S_{1}\right)$ of the system being in overload mode is equal to the sum of the corresponding probabilities, i.e.,

$$
P\left(S_{1}\right)=\sum_{(j, s) \in S_{1}} p_{j, s}=\sum_{j=L}^{R} p_{j, 1} .
$$

Similarly, one can find the stationary probability $P\left(S_{2}\right)$ of the system being in discard mode. We have

$$
P\left(S_{2}\right)=\sum_{(j, s) \in S_{2}} p_{j, s}=\sum_{j=H+1}^{R-1} p_{j, 2} .
$$

Finally, the stationary probability $P\left(S_{0}\right)$ of the system being in normal mode is

$$
P\left(S_{0}\right)=\sum_{(j, s) \in S_{0}} p_{j, s}=\sum_{j=0}^{H-2} p_{j, 0} .
$$

As mentioned in the previous section, the mean control cycle time is inverse to the stationary intensity of the instants of control cycle starts. Since the control cycle starts when the system passes from state $(L, 1)$ to state $(L-1,0)$, the stationary intensity of instants of control cycle starts is equal to the stationary intensity of passes from state $(L, 1)$ to state $(L-1,0)$ :

$$
\mu=\nu q_{L, 1} \int_{0}^{\infty} e^{-\lambda_{1} x} \mathrm{~d} B(x)=\nu \beta_{0}^{1} q_{L, 1} .
$$

Hence

$$
\tau=\mu^{-1}=\frac{1}{\nu \beta_{0}^{1} q_{L, 1}} .
$$

Thus the mean time $\tau_{12}$ that the system spends in overload and the discard set of states during one control cycle can be calculated by the formula (52).

\section{Numerical example}

In this section we present results of computation of the mean return time of the system from overload mode to normal load mode. Two policies, P1 and P2, were compared under the exponential service time and the constant service time with the same mean value of $b=1$. The values of the thresholds were set to $L=8, H=12$, $R=20$. The distribution of the number of customers in the batch was assumed to be uniform, i.e., $\omega_{k, n}=0.2$, where $k=1,2$ and $n=\overline{1,5}$; the input flow rate $\lambda=2 / 3$. Since the average number of customers in the batch is equal to 3 , the offered load is $\rho=2$.

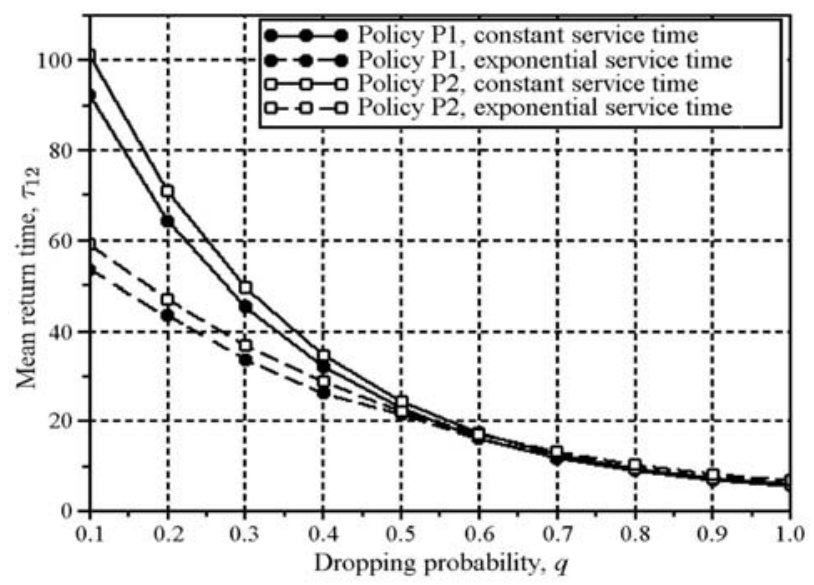

Fig. 2. Mean return time $\tau_{12}$ dropping probability $q$ for the two policies of overload control.

Figure 2 shows the dependence of mean return time $\tau_{12}$ on the dropping probability $q$ for the two policies. The first thing to notice is that the mean return time for an 
exponential service time is less than those for a constant time for the values of $q<0.6$ for both policies. At the same time the mean return time, for policy $\mathrm{P} 1$ is always less than those for policy P2. It is predictable since policy $\mathrm{P} 1$ is applied at all possible instants of the queue-length change. As might be expected, the computations showed that the expectation of $\tau_{0}$ of the system being in the set of normal load is independent of the dropping probability $q$. The time the system spends in normal mode for policy P2 is always greater than for policy P1.

\section{Conclusion}

This paper provides methods for stationary analysis of the queueing system with batch arrivals and hysteretic overload control policy modeling an SIP-server with a load control mechanism. There were obtained key performance characteristics of the system related to the number of customers in it and the control policy (namely, the mean time between changes of the system's mode). Analysis was carried out under two assumptions: a change in the system mode happens each time the number of customers in the system changes; a change in the system mode happens only at consecutive service completion epochs. Numerical analysis shows that hysteretic policy under both assumptions has features beneficial to SIP-server overload control. The choice of the assumption for practical implementation is the subject of further study and requires formulation and solution of the optimization problem.

\section{Acknowledgment}

This work was supported in part by the Russian Foundation for Basic Research (grants 12-07-00108, 13-07-00665, and 13-07-00953).

\section{References}

Abaev, P., Gaidamaka, Y. and Samouylov, K. (2011). Signaling load hysteretic control in the SIP-servers network, Bulletin of Peoples' Friendship University of Russia: Mathematics, Informatics, Physics 1(4): 54-71.

Abaev, P., Gaidamaka, Y. and Samouylov, K. (2012a). Queuing model for loss-based overload control in a SIP server using a hysteretic technique, in S. Andreev, S. Balandin and Y. Koucheryavy (Eds.), Internet of Things, Smart Spaces, and Next Generation Networking, Springer-Verlag, Berlin, pp. 371-378.

Abaev, P.O., Gaidamaka, Y.V., Pechinkin, A.V., Razumchik, R.V. and Shorgin, S.Y. (2012b). Simulation of overload control in SIP server networks, Proceedings of the 26th European Conference on Modelling and Simulation, ECMS 2012, Koblenz, Germany, pp. 533-539.

Abaev, P., Gaidamaka, Y., Samouylov, K., Pechinkin, A., Razumchik, R. and Shorgin, S. (2014). Hysteretic control technique for overload problem solution in network of SIP servers, Computing and Informatics 33(1): 1-18.

Bekker, R. (2009). Queues with levy input and hysteretic control, Queueing Systems 63(1): 281-299.

Benaboud, H. and Mikou, N. (2002). Analysis by queuing model of multi-threshold mechanism in ATM switches, Proceedings of the 5th IEEE International Conference on High Speed Networks and Multimedia Communications, HSNMC 2002, Jeju Island, Korea, pp. 147-151.

Bocharov, P.P., D‘Apice, C., Pechinkin, A.V. and Salerno, S. (2004). Queueing Theory, VSP, Utrecht/Boston, MA.

Brown, P., Chemouil, P. and Delosme, B. (1984). A congestion control policy for signalling networks, Proceedings of the 7th International Conference on Computer Communications, ICCC 1984, Sydney, Australia, pp. 717-724.

Dshalalow, J.H. (1997). Queueing systems with state dependent parameters, in J. Dshalalow (Ed.), Frontiers in Queueing: Models and Applications in Science and Engineering, CRC Press, Boca Raton, FL, pp. 61-116.

Gebhart, R.F. (1967). A queuing process with bilevel hysteretic service-rate control, Naval Research Logistics Quarterly 14(1): 55-68.

Golubchik, L. and Lui, J.C.S. (1997). Bounding of performance measures for a threshold-based queueing system with hysteresis, Proceedings of the ACM SIGMETRICS Conference, ACC 2001, Seattle, WA, USA, pp. 147-157.

Hilt, V., Noel, E., Shen, C. and Abdelal, A. (2011). Design considerations for session initiation protocol (SIP) overload control, Technical Report RFC-6357, Internet Engineering Task Force.

Kaczorek, T. (2013). Minimum energy control of positive continuous-time linear systems with bounded inputs, International Journal of Applied Mathematics and Computational Science 23(4): 725-730, DOI: 10.2478/amcs-2013-0054.

Krasnoselskii, M.A. and Pokrovskii, A.V. (1989). Systems with Hysteresis, Springer-Verlag, Berlin/Heidelberg.

Moltchanov, D., Koucheryavy, Y. and Harju, J. (2006). Loss performance model for wireless channels with autocorrelated arrivals and losses, Computer Communications 29(13-14): 2646-2660.

Olwal, T.O., Djouani, K., Kogeda, O.P. and van Wyk, B.J. (2012). Joint queue-perturbed and weakly coupled power control for wireless backbone networks, International Journal of Applied Mathematics and Computational Science 22(3): 749-764, DOI: 10.2478/v10006-012-0056-z.

Pechinkin, A.V., Razumchik, R.V. (2013a). Approach for analysis of $M_{2}\left|M_{2}\right| 1 \mid R$ with hysteric policy for SIP server hop-by-hop load control, Proceedings of the 27th European Conference on Modelling and Simulation, ECMS 2013, Alesund, Norway, pp. 573-579.

Pechinkin, A.V., Razumchik, R.V. (2013b). Stationary distribution of $M_{2}|G| 1 \mid r$ queue with bi-level hysteric policy, Journal of Communications Technology and Electronics 58(12): 1220-1229. 
Roughan, M. and Pearce, C. (2000). A martingale analysis of hysteretic overload control, Advances in Performance Analysis: A Journal of Teletraffic Theory and Performance Analysis of Communication Systems and Networks 3(1): $1-30$.

Russell, T. (2006). Signaling System \#7, 5th Edition, McGraw-Hill, New York, NY.

Sindal, R. and Tokekar, S. (2008). Modeling and analysis of voice/data call admission control scheme in CDMA cellular network for variation in soft handoff threshold parameters, Proceedings of the 16th IEEE International Conference on Networks, ICON 2008, New Delhi, India, pp. 1-6.

Takagi, H. (1985). Analysis of a finite-capacity $M / G / 1$ queue with a resume level, Performance Evaluation 5(3): 197-203.

Takshing, P. Y. and Yen, H.-M. (1983). Design algorithm for a hysteresis buffer congestion control strategy, Proceedings of the IEEE International Conference on Communications, Boston, MA, USA, pp. 499-503.

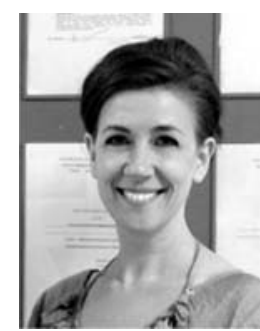

Yuliya V. Gaidamaka received her Ph.D. in mathematics from the Peoples' Friendship University of Russia in 2001. Since then, she has been an associate professor, first at the university's Telecommunication Systems Department, then at the Applied Probability and Informatics Department. She is the author of more than 50 scientific and conference papers. Her research interests include SIP signaling, multiservice and OFDMA based networks. P2P network performance analysis, as well as

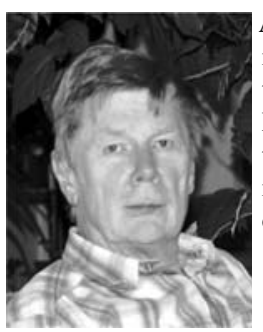

Alexander V. Pechinkin is a Doctor of Sciences in physics and mathematics and a principal scientist at the Institute of Informatics Problems of the Russian Academy of Sciences, and a professor at the Peoples' Friendship University of Russia. He is the author of more than 200 papers in the field of applied probability theory.

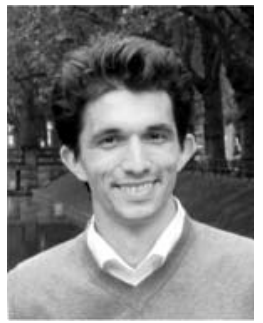

Rostislav V. Razumchik received his Ph.D. in physics and mathematics in 2011. Since then, he has worked as a senior researcher at the Institute of Informatics Problems of the Russian Academy of Sciences and a senior lecturer at the Peoples' Friendship University of Russia. His current research activities focus on queueing theory and related simulation issues.

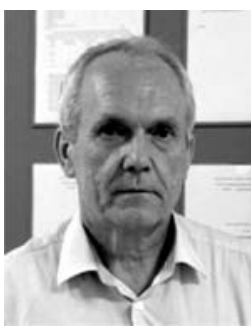

Konstantin E. Samouylov, a professor, received his $\mathrm{Ph} . \mathrm{D}$. degree in probability and queueing theory from the Moscow State University in 1985 and the D.Sc. degree from the Moscow Technical University of Communications and Informatics in 2005. During the last two decades he has held several positions at the Peoples' Friendship University of Russia, where in 1996 he became the head of the Telecommunication Systems Department, and in 2014 the head of the Applied Probability and Informatics Department. He has done research for the Helsinki and Lappeenranta Universities of Technology, several institutes of the Russian Academy of Sciences, and a number of Russian telecom operators. His current research interests are performance analysis of next generation networks, teletraffic and queueing theory. He has written more than 100 scientific and technical papers, and three books.

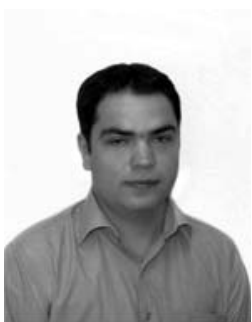

Eduard Sopin received a Ph.D. in mathematics from the Peoples' Friendship University of Russia in 2014. Since then, he has been a senior lecturer at the university's Applied Probability and Informatics Department. His research interests include queueing theory and SIP signaling.

Received: 19 August 2013

Revised: 26 February 2014 